\title{
LOS MANUALES DE LECTURA, UN PATRIMONIO DE LA HISTORIA EDUCATIVA DE MEXICO
}

\author{
Lucía Martínez Moctezuma \\ Universidad Autónoma del Estado de Morelos/México \\ luciamm@uaem.mx
}

\begin{abstract}
RESUMEN
El objetivo de este trabajo es reflexionar sobre los métodos de lectura que circularon a través de una serie de manuales escolares. Se trata de textos que forman parte de la Biblioteca Escolar resguardada en la Universidad Autónoma del Estado de Morelos (México) pero también del patrimonio histórico educativo de nuestro país. Analizaremos particularmente textos que sirvieron para la enseñanza de la lectura escritura y que, bajo un modelo similar circularon en América Latina. En esta vertiente, los libros de texto, se convierten en un objeto de investigación privilegiada para conocer la práctica educativa pues desde el origen de los sistemas nacionales en el siglo XIX, ocuparon un lugar privilegiado en el aula. Sus contenidos, su elaboración, su formato y su edición, los convierten en una fuente primordial para entender la historia interna de la escuela.
\end{abstract}

Palabras clave: Biblioteca Escolar. Libros de texto. Enseñanza de la lectura.

\section{THE READING TEXTBOOK, A HERITAGE OF THE HISTORICAL EDUCATION OF MEXICO}

\begin{abstract}
The aim of this paper is to reflect on reading methods that circulated through a series of textbooks. These are texts that are part of not only sheltered School Library at the Universitidad Autonoma del Estado de Morelos (Mexico) but the educational heritage of our country. Particularly we analyze texts which served for teaching reading and writing, under a similar model circulated in Latin America. In this aspect, textbooks, become an object of investigation privileged to learn from educational practice as the source of national systems in the nineteenth century, occupied a privileged place in the classroom. Contents, preparation, format and editing, make them a key to understanding the internal school history source.
\end{abstract}

Keywords: School Library. Textbooks. Teaching reading.

\section{INTRODUCCIÓN}

Gran parte de los libros que sirvieron para el aprendizaje de la lectura y escritura de los niños latinoamericanos llegaron de Francia, España y Estados Unidos. A finales del siglo XIX y primeras décadas del XX, fueron traducidos y adaptados al castellano, inspirando a los profesores 
a escribir libros con contenidos más próximos a lo que la elite educativa esperaba de sus ciudadanos. En estas preocupaciones es importante subrayar que para la escritura de los textos no se contemplaron las diferencias multilingües.

Amén de algunos textos escritos en lengua indígena ${ }^{1}$, no hubo realmente una preocupación de los Estados por reconocer las diferencias y las necesidades de esta población que, paradójicamente tampoco estaba registrada de manera cuantitativa ${ }^{2}$. En México si bien en el período independiente (1810-1821) una parte de la población fue considerada indígena, hablante de lenguas indígenas, años después, a partir del período del Presidente Porfirio Díaz y la Revolución Mexicana (1876-1910), una mayoría de la población fue considerada mestiza, rural, hablante del español, con una minoría indígena, que para fines educativos representaba alrededor de 11 millones de indígenas y mestizos frente a un millón y medio de blancos, mereció las reflexiones de Moisés Saenz, "Nuestra frase de [...] incorporar el indio a la civilización" debería ser cambiada por la de "Incorporar la civilización al indio" y dejar que, asimilándola, la transforme en la que al fin y al cabo será la civilización mexicana..."3

En este trabajo me interesa reflexionar sobre los métodos que sirvieron para estos fines. Se trata de textos que forman parte de la Biblioteca Escolar que ahora está resguardada en la Universidad Autónoma del Estado de Morelos y que forma parte del patrimonio histórico educativo de nuestro país. Analizaremos particularmente textos que sirvieron para la enseñanza de la lectura escritura y que, bajo el mismo modelo circularon en América Latina. En esta vertiente, los libros de texto, se convierten en un objeto de investigación privilegiada para conocer la práctica educativa pues desde el origen de los sistemas nacionales en el siglo XIX, ocuparon un lugar privilegiado en el aula. Sus contenidos, su elaboración, su formato y su edición, los convierten en una fuente primordial para entender la historia interna de la escuela.

\footnotetext{
${ }^{1}$ Dorothy Thanck considera que entre 1784 y 1821, se publico el primer libro de texto gratuito en México financiado por el gobierno indígena de la capital y distribuido gratuitamente a los estudiantes de las escuelas de las parcialidades, donde se enseñaba el catecismo, los primeros pasos de la lectura en nahuatl y gradualmente la enseñanza del castellano. Tanck de Estrada, 2011, p. 53-64.

${ }^{2}$ Los misioneros habían buscado catequizar a los indígenas en sus lenguas (de ahí la publicación de catecismos y gramáticas indígenas). El recurso a la lengua «nacional » fue desde 1821, un rasgo de la escuela pública. Los dos grandes presidentes del siglo XIX, Benito Juárez y Porfirio Díaz, tuvieron origen indígena. Véase como ejemplo Bravo Ahuja Gloria, 1977, p. 22-55.

${ }^{3}$ Moisés Sáenz, 1931, p. 445.
} 


\section{PRESCRIPCIONES PARA LA ENSEÑANZA DE LA LECTURA}

Una de las resoluciones más importantes en América Latina fue erradicar el método mutuo, que si bien permitía instruir a una gran cantidad de niños, debido al apoyo de alumnos con conocimientos más avanzados, sólo estimulaba la repetición y la memoria. En su lugar se acordó el método simultáneo que cumplía con los fines instructivos y educativos; se aconsejó formar grupos de un máximo de 50 alumnos e impartir clases orales.

Para la enseñanza simultánea de la lectura y escritura (impuesta en Europa en los años 1860 y en México, a partir de los congresos de instrucción pública entre 1889-1890) ${ }^{4}$, se adoptó el uso de alguno de los tres sistemas de enseñanza entonces en boga: deletreo, silabeo y fonetismo, con los que se distinguían los métodos básicos para la enseñanza del sistema analíticosintético o sintético-analítico como se ve en los ejemplos de Guyau (1892); Carlos A. Carrillo (1904) y Ricardo Gómez (1907), así como en los dos más populares de la época en México: el Método de escritura lectura de Enrique C. Rebsamen (1899) y el Método Onomatopéyico para enseñar a leer y escribir simultáneamente de Gregorio Torres Quintero (1904) 5 .

El Nuevo método inductivo, analítico, sintético para la enseñanza simultanea de la lectura y escritura de Carlos A. Carrillo se dividía en tres partes: oral (la pronunciación correcta de la palabra); escrita y técnica (los conocimientos gramaticales). La lectura se enseñaba al mismo tiempo que la escritura. Su argumento se basaba en el hecho de que para memorizar una letra, el niño debía copiarla, examinarla y compararla con la que él escribía. A fuerza de tanto repetir, se aprendía la forma de la letra diferenciando entre la de imprenta y la manuscrita. Carrillo recomendaba el texto de Claudio Matte, chileno y discípulo de Klauwell, quien había instituido un estudio preparatorio para enseñar a leer asociando las figuras de 27 letras con 21 sonidos diferentes, de tal manera que viendo la letra se imitaba el sonido. Antes de conocer la letra había que hacer el análisis auditivo de la palabra. Carrillo se decía partidario del deletreo

\footnotetext{
${ }^{4}$ La cuestión de las prácticas en la historia de la educación, sigue siendo un tema que preocupa a los investigadores. Para abordar los métodos de lectura, se ha recurrido a una serie de fuentes (leyes, oficios, programas, libros de texto y memorias de maestros y alumnos sobre todo para el siglo XX) que si bien han resultado limitadas para entender las prácticas dentro de la escuela, han inspirado buenos ejemplos de la historiografía de la educación en México. Dorothy Tanck ha sugerido prestar atención a los útiles escolares para un acercamiento mayor a la vida cotidiana; Luz Elena Galván, a los periódicos infantiles para acercarse a la práctica de la lectura y Elsie Rockwell, estudiar la cultura escolar del pasado con una mirada etnográfica. Civera Alicia, Escalante Carlos y Galván Luz Elena, 2002, p. 184-234.

${ }^{5}$ Hay apenas una generación de diferencia con Europa, esto no significa que en 1900 todos los alumnos que ingresaban por primera vez a la escuela tuvieran el material necesario para aprender a escribir en cada clase ( un pizarrón, una pluma y un cuaderno).
} 
perfeccionado y no del método fonético, es decir, proponía la sustitución de los nombres de algunas letras por otros que facilitaran la lectura y, deletrear, en seguida las palabras que se querían leer designando las letras por sus nombres, por ejemplo, rosa, se deletreaba como: re o, ro; se, a, sa: rosa.

Otros textos como el ler Año del Lector Hispanoamericano de Ricardo Gómez o El Año Preparatorio de Lectura Corriente de M. Guyau (editado en 1892 por la Librería Armand Colin de Francia,), fueron desestimados por la Junta Académica de la Escuela Normal de Veracruz con el argumento de que si bien se ejercitaba la división de palabras en sílabas y esto simplificaba el esfuerzo mental de los niños, sus ejemplos atendían a una realidad Europea desconocida para los niños mexicanos. Otros textos mexicanos se inspiraron de autores chilenos como Claudio Matte. Lo vemos en el caso del texto mexicano de Ricardo Gómez.

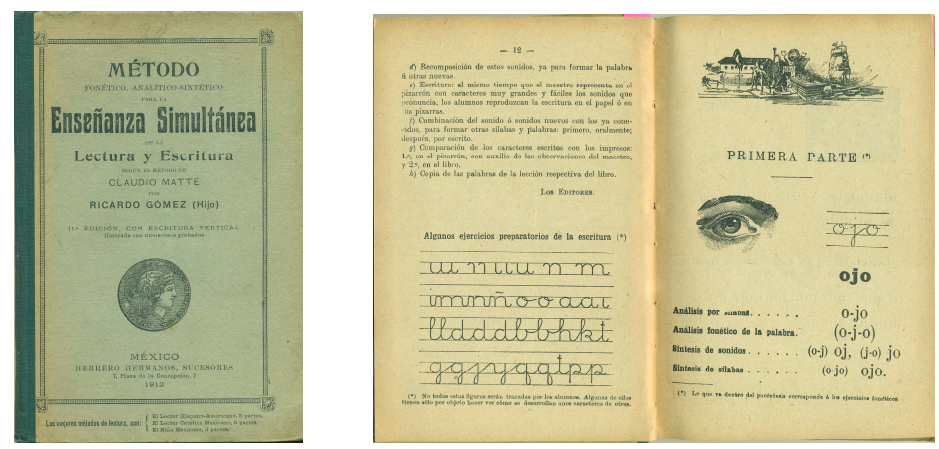

Fuente: BEUAEM. Gómez Ricardo (1913) Método de Enseñanza simultanea de la lectura y escritura. México: Herrero Hnos.

Amén de este ejemplo hubo otros método de lectura como los del suizo Enrique Rebsamen y el de su discípulo mexicano, Gregorio Torres Quintero cuyo ejemplo será seguido en países como Cuba.
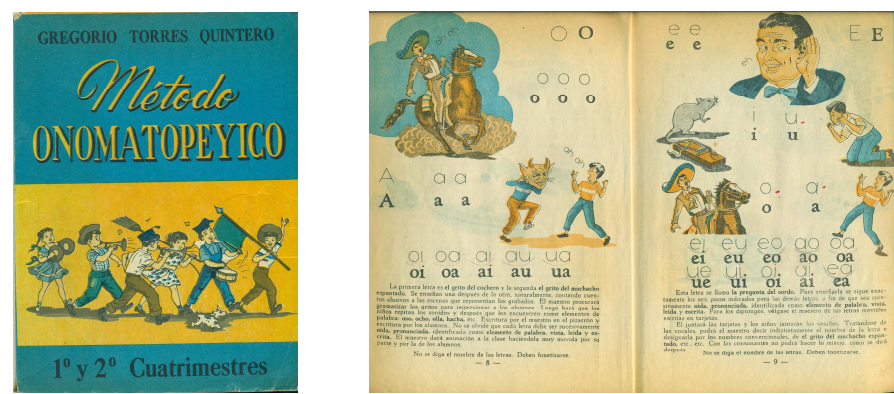

Fuente: BEUAEM. Torres Quintero Gregorio (1970). Método Onomatopeyico. México (a partir de la versión original de 1904) 


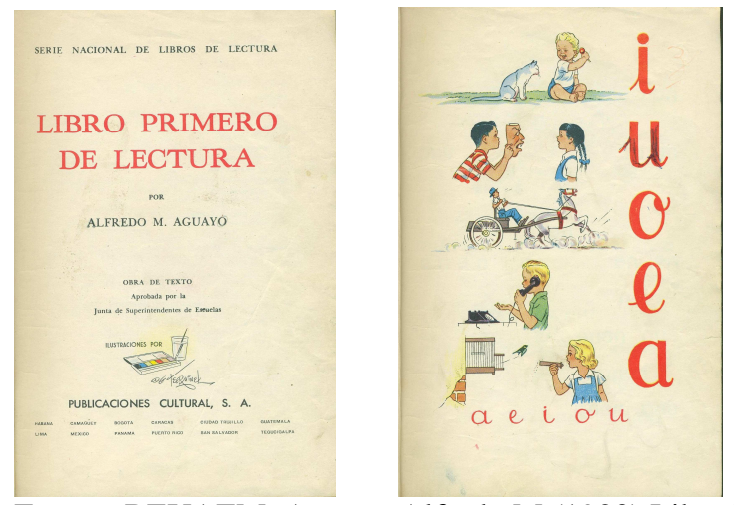

Fuente: BEUAEM. Aguayo Alfredo M (1920) Libro Primero de Lectura. Cuba: Publicación Cultural SA.

\section{LOS MÉTODOS}

Enrique Conrado Rebsamen ${ }^{6}$ introdujo el método de palabras normales en la Escuela Anexa a la Normal de Veracruz en 1886. El método consistía en enseñar cada palabra como un todo, sin estudio previo de sus elementos fonéticos, donde se asociaba la forma gráfica de la palabra con su significado. Se enseñaba la lectura a partir de la escritura bajo tres principios pedagógicos: simultaneidad, fonetismo y marcha analítico-sintética mediante "palabras normales": simultaneo porque enseñaba a leer y escribir al mismo tiempo (primero, los caracteres manuscritos y luego los impresos); fonético porque a diferencia del deletreo, familiarizaba a los niños con los sonidos y analítico-sintético porque seguía un orden en el que se presentaban primero la palabra, después la división en sílabas y por último las letras representadas por sus sonidos, para regresar a las sílabas y luego a la palabra.

En su Guía Metodológica de la Enseñanza de la Escritura y Lectura (1899), Rebsamen señala que su Método de Palabras Normales (1898) se basaba en el de los alemanes Carlos Vogel, M.O.Kramer y Juan Bautista Graser, perfeccionado por Adolfo Klauwell, introducido en Chile por Claudio Matte y en México por José Manuel Guillé (1877) ${ }^{7}$. En estos textos se planteó por primera vez el uso de los ejercicios intuitivos en el desarrollo del lenguaje: ver, hablar y

\footnotetext{
${ }^{6}$ Enrique Conrado Rebsamen Egoff (Kreuzlingen, Suiza 1857-Veracruz, México 1904), se graduó en la Escuela Normal de Kuznacht, Zurich, en 1874. Llegó a México en 1883 por contacto con Caros Von Gagern, militar alemán y Ministro de Obras en 1861. Su red de relaciones se amplió a Ignacio Altamirano, director de la Escuela Normal y Juan Luz Enríquez, gobernador de Veracruz, quienes lo incorporaron al proyecto de la Escuela Modelo de Orizaba. En los dos congresos de instrucción pública mexicanos (1889-1890) se encargó de introducir a la discusión las propuestas del francés Ferdinand Buisson y del español Pedro Alcántara. Fue director de la Escuela Normal de Jalapa, Veracruz y de su Revista México Intelectual (1889-1904). Colaboró con el proyecto educativo de entidades como Oaxaca (1891), Jalisco (1892) y Guanajuato (1893-1895). Zollinger Edwin, 1957.
}

${ }^{7}$ Guillé Manuel, 1877. 
escribir. Para conocer los objetos era primordial la observación y el dibujo, después venía el aprendizaje de palabras completas, asociando la imagen y la letra a los sonidos (por ejemplo, la "s" para la silbadora, la "r" para la matraca).

El método marcaba cuatro etapas distribuidas en dos partes y varias subdivisiones: los ejercicios preparatorios, la enseñanza de las vocales, la escritura-lectura de las letras mayúsculas y minúsculas y la lectura de las letras impresas ${ }^{8}$. A partir del segundo año se familiarizaba a los niños con todos los sonidos y se iniciaba la enseñanza de las letras por medio de palabras normales; muy pocas correspondían al medio rural (yunta, zopilote, jacal) pero en general todas atendían al universo urbano (tina, cama, soldado, muñeca). Para fortalecer la memoria visual y auditiva del niño se hacían ejercicios de copia y dictado. Se enseñaba una letra por semana y hasta el cuarto grado, los maestros hacían uso del alfabeticón o máquina de lectura, que consistía en una serie de letras móviles para formar palabras y frases, también había una caja tipográfica para que los alumnos formaran las frases que leían.

\section{b. El método fonético para la escritura de Torres Quintero}

Al igual que Rebsamen, Gregorio Torres Quintero escribió un Método (1904) y una Guía del Método Onomatopéyico (1908). Su propuesta se definía como fonética porque empleaba los sonidos de las letras y no sus nombres. Su fonetismo era onomatopéyico porque el sonido de las letras se obtenía de la imitación fonética de los ruidos y las voces. Era sintético porque partía de los sonidos para formar sílabas, palabras y luego frases. Analítico, porque en sus ejercicios orales las palabras se descomponían en sílabas. Simultáneo porque asociaba la lectura con la escritura. Una vez que el niño sabía leer y escribir con letra manuscrita, empezaba con los caracteres impresos 9 .

Torres Quintero afirmaba haberse inspirado en Comenio y Agustín Grosselin, creador del método fonomímico para sordomudos que el autor conoció en las clases de su Profesor Rafael Izunza de la Escuela Normal de la Ciudad de México. La diferencia con la fononimia de Grosselin se encontraba en que el sonido (la letra) se acompañaba de un gesto mientras que la onomatopeya,

${ }^{8}$ Barbosa Heldt Antonio, 1971, p. 35-47.

${ }^{9}$ Ibid., 48-69.

Rev. Iberoam. Patrim. Histórico-Educativo, Campinas (SP), v. 1, n. 1, p. 37-47, jul./dez. 2015 
asociaba sonido-imagen, a diferencia de lo que se hacía antes, cuando se pedía sólo atención a algún detalle de la letra impresa.

El autor señalaba como una ventaja de su método que el progreso en la lectura era gradual tanto en la lectura oral como silenciosa y en velocidad como en comprensión. Partía de una idea central: el niño debía aprender a leer como había aprendido a hablar, es decir, por frases, porque era natural para él, la vocalización, es decir el ensayo lingüístico. Según Torres Quintero cuando el niño se preparaba para hablar, había un periodo preparatorio del lenguaje en el que no intervenía el pensamiento. Al vocalizar, el niño ensayaba en un sonido y en una articulación, una sílaba, al terminar iniciaba otro ejercicio. En este mecanismo de la lectura debían funcionar los elementos de la palabra, es decir, los sonidos y las sílabas, que al repetir en su gimnasia vocal, en su juego lingüístico conducían a la pronunciación de la palabra. Tal cual debía iniciar el aprendizaje de la lectura ${ }^{10}$

\section{ALFABETIZAR PARA ASIMILAR: LA ESCOLARIZACIÓN RURAL}

Con los primeros brotes de la Revolución Mexicana (1911-1917), otros proyectos educativos tomarán cauce. La Ley del 30 de mayo de 1911, planteaba la organización de las escuelas de instrucción rudimentaria cuyo proyecto fue elaborado por el propio Gregorio Torres Quintero, quien fue Jefe de la Sección de Instrucción Rudimentaria del Ministerio de Instrucción Pública. La formación, en dos cursos anuales y sin carácter obligatorio, estaba destinada principalmente a los individuos de raza indígena para enseñarles a hablar, leer y escribir en español así como ejercitarlos en las cuatro operaciones aritméticas. Para estimular la asistencia de los alumnos se propuso la distribución de alimento y vestido, según las circunstancias. Como en otra época, Torres Quintero defendía la idea de que la enseñanza del español debía ser directa, como en el aprendizaje de la lengua materna o en el de una lengua extranjera, como en el método Berlitz, donde el profesor ignoraba la lengua de sus alumnos, para evitar la traducción y estimular la transmisión directa ${ }^{11}$.

Con poco éxito, las escuelas rudimentarias quedaron en el olvido. Finalizado el movimiento armado, la recién creada Secretaría de Educación Pública (1921) retomó la idea de

\footnotetext{
${ }^{10}$ Torres Quintero Gregorio, 1929, p. 8-33.

${ }^{11}$ Torres Quintero Gregorio, 1913.
} 
“incorporar a la civilización” a los 10324484 millones de mexicanos que aún no sabía leer ni escribir (3 615320 en edad escolar y 6709164 adultos) ${ }^{12}$. A través de su Departamento de Escuelas Rurales e Incorporación Cultural Indígena, las Misiones Culturales, emprendieron su labor mas allá de la escuela pues como se había evidenciado en el proyecto de Torres Quintero, el número de lenguas que se hablaba seguía siendo cuantioso pero indeterminado (entre 183 y 204 aproximadamente).

Moisés Sáenz subsecretario de educación (1925-1930) y Rafael Ramírez director de este Departamento hasta 1934, adoptaron el proyecto de la incorporación del indígena a la sociedad como el mejor medio para resolver el problema de la heterogeneidad racial y cultural del país. La homogeneización fue el requisito para la construcción del orden social: una escuela democrática, en la que convivieran indios y mestizos dentro de sus comunidades ${ }^{13}$. El año rudimentario de las Casas del Pueblo cambio su nombre por preparatorio, aunque en el fondo el objetivo siguió siendo el mismo: enseñar español a los niños indígenas. Esta tarea se amplió a toda la comunidad por lo que dar al pueblo un idioma común fue uno de los objetivos de la Secretaría de Educación Pública. Moisés Saenz; inició una cruzada a favor de la castellanización, lanzando una convocatoria para encontrar un método de fácil aplicación en el campo. Respondieron algunos maestros de escuelas primarias, secundarias y de idiomas como inglés. Ramírez resumió la posición oficial para servir de guía: aconsejaba la castellanización directa, sin traducción, retomando las ideas de Torres Quintero en general, la metodología de Ramírez recogía muchas de las experiencias cotidianas y sugerencias de los maestros: labores agrícolas, cuidado de los animales domésticos, trabajos manuales, formación de un pequeño museo escolar, relatos de cuentos y leyendas incluyendo las "de diferentes razas indígenas que poblaron nuestro suelo...". Para el aprendizaje del castellano, recomendaba al profesor que reflexionara sobre ese método natural y sencillo que las mamas empleaban para enseñar a hablar a sus hijos, desde que “...les entonan los primeros ritmos, los entretienen con los primeros juegos y los encantan con los primeros cuentos...”. Las respuestas quedaron condensadas en el libro de Rafael Ramírez, Como dar a Mexico un idioma.

\footnotetext{
${ }^{12}$ Galván Luz Elena, 1985: 31. De los casi 15 millones de habitantes del país, 4200000 estaban clasificados como indios y alrededor de 8 millones como "gente cuyo nivel de vida es primitivo". Ramírez Rafael, 1976, p. 10.

${ }_{13}$ Tanto Ramírez como Sáenz egresan de la Escuela Normal de Veracruz. Sáenz estudia además un posgrado en la Universidad de Columbia donde conoce las propuestas de John Dewey y defiende una tesis sobre la escuela secundaria. Los dos comparten proyectos con Torres Quintero.
} 


\section{RECOMENDACIONES Y USOS DEL “MÉTODO NATURAL"}

En su propuesta, Rafael Ramírez recomendó a los maestros el uso del método natural y el texto Rie escrito por Alfredo Uruchurtu y Ernesto Alconedo ${ }^{14}$. En éste se encontraba una serie de argumentos de orden físico y mental que atendían a la coordinación y precisión para el ejercicio de la lectura de cuentos, siempre que estuvieran impresos. Teniendo en cuenta que el niño aprendía en el hogar los giros y las palabras difíciles del idioma, guiado por el interés o la necesidad, la propuesta de Uruchurtu y Alconeda- suprimía el análisis de las palabras para leer frases cuya temática se orientaba al universo del alumno, otra vez, del medio urbano: el hogar, la calle y la escuela. La enseñanza de la lectura se iniciaba con el relato de un cuento cuyo contenido servía para seleccionar frases completas que se ejercitaban en lecciones orales, dramatizaciones y lectura en voz alta.

Torres Quintero fue uno de los principales críticos de este método porque subrayó el hecho de que los niños leían por conjuntos (frases) pero sin análisis ni síntesis. En su réplica quedaron de lado las objeciones de autores como Dean Kappler, de la Escuela de Educación del Colegio de la ciudad de Nueva York, quien veía como un impedimento el hecho de que los alumnos memorizaran el cuento, siguiendo la lectura frase por frase y con el aprendizaje de palabras fuera de contexto. El resultado final parecía ser el recuerdo de un cuento reducido a partículas donde se perdía lo vital y sobre todo lo sugestivo de la lectura. Para Torres Quintero, el método del cuento era apropiado para el idioma inglés que no era fonético; para el español, que si era fonético, se requería de otros métodos.

\section{NOTAS FINALES}

Aprender el castellano fue seguido de otros elementos básicos de la cultura enseñados por profesores mestizos, que desconocían una serie de elementos culturales para entender la región en la que laboraban. Los contenidos educativos, al no estar vinculados con las características del entorno, propiciaron la memorización de palabras, conceptos e ideas; si los niños siguieron

\footnotetext{
${ }^{14}$ Uruchurtu Alfredo y Alconedo Ernesto, 1928: 143-150. Uruchurtu fue Jefe del Departamento de Enseñanza Agrícola y Normal en 1927. Las imágenes de este libro fueron realizadas por German Gedovius (ciudad de México, 1867-1937) quien realizó estudios en la Academia de San Carlos y en 1887, se especializó en técnicas pictóricas y dibujo al carbón en la Academia de Bellas Artes en Alemania
} 
hablando una lengua que los maestros no entendían, aprendieron el castellano repitiendo palabras, leyendo frases y desconociendo su significado. Vistas las dificultades en el medio rural trató de usarse sobre todo "el sentido común", para enseñar a leer y luego a escribir, o viceversa, en dos momentos porque siguieron considerándose como dos habilidades diferentes.

Hasta antes de 1940, la enseñanza del español en Latinoamérica ha ido a un ritmo muy lento, con titubeos, pocos aciertos y dudosos resultados. Los proyectos podrían considerarse como lo observaba el lector anónimo que me antecedió en la lectura de la Enseñanza del Lenguaje de Rafael Ramírez, quien dejó sus observaciones en la última página del texto en noviembre de 1966: “....aunque pedagógicamente es muy bueno... este libro....contiene sólo un regular conjunto de buenas indicaciones...”. Prescripciones que los maestros de la zona rural encontraron en los métodos de lectura, que tomaron como sugerencias, como un conjunto de buenas indicaciones, porque se enfrentaron al uso de una lengua que de manera frecuente no entendían y que no fue considerada ni en los métodos ni en los libros escolares.

\section{BIBLIOGRAFÍA}

BARBOSA HELDT, Antonio. Cómo han aprendido a leer y escribir los mexicanos. México: Editorial Pax México, 1971, p. 35-47.

BEUAEM Biblioteca Escolar. Universidad Autónoma del Estado de Morelos.

BRAVO AHUJA, Gloria. La enseñanza del español a los indígenas mexicanos. México: El Colegio de México, 1977, p. 22-55

CIVERA, Alicia; ESCALANTE, Carlos y GALVAN LUZ, Elena (coords). Debates y desafíos en la historia de la educación en México. México: El Colegio Mexiquense, ISCEEM, 2002, p. 184-234.

GALVÁN LUZ, Elena. Los maestros y la educación pública en México. Un estudio histórico. México: CIESAS, 1985.

GUILLÉ, Manuel. La enseñanza elemental. Guía teórica-práctica para la instrucción primaria en la enseñanza objetiva. Veracruz: Imprenta del Estado, 1877.

Los Maestros y la Cultura Nacional, 1920-1952. México: Museo Nacional de las Culturas Populares, Dirección General de Culturas Populares, SEP.

RAMÍREZ, Rafael. La escuela rural mexicana. México: SEP SETENTAS, 1976, p. 10.

TANCK DE ESTRADA, Dorothy. "El primer libro de texto gratuito en México: la biografía de una mujer indígena publicada en 1874" en Barriga Villanueva Rebeca (coord). Entre paradojas: 
a 50 años de los Libros de Texto Gratuitos. México: El Colegio de México, SEP, Conaliteg, 2011, p. 53-64.

TORRES QUINTERO, Gregorio. Los métodos de lectura americanos. Su inadaptabilidad al español. Discusión de las resoluciones relativas a la enseñanza de la lectura en el primer año elemental aprobadas por la Conferencia Pro-Lengua Nacional de marzo 1928. México: Imprenta Labor, 1929.

La instrucción rudimentaria en la República. Estudio presentado en el Primer Congreso Científico Mexicano. México Imprenta del Museo Nacional de Arqueología, Historie y Etnología, 1913.

URUCHURTU, Alfredo y ALCONEDO, Ernesto. Rie. Método de Lectura y Escritura para el primer Año por los profesores... México: Sociedad de Edición y Librería Franco Americana SA, 1928.

ZOLLINGER, Edwin. Enrique C.Rebsamen. El renovador de la instrucción primaria en México. México: Ediciones de la Dirección General de Educación del Estado de Veracruz, 1957. 